\title{
Automated Peritoneal Dialysis: Patient Perspectives and Outcomes
}

\author{
Alessandro Domenici' \\ Anna Giuliani ${ }^{2}$ \\ 'Department of Clinical and Molecular \\ Medicine, "Sapienza" University, \\ Sant'Andrea Hospital, Nephrology and \\ Dialysis Unit, Rome, Italy; ${ }^{2}$ Department \\ of Nephrology Dialysis and \\ Transplantation, San Bortolo Hospital, \\ Vicenza, Italy
}

\begin{abstract}
A steadily increasing number of end stage kidney disease (ESKD) patients are maintained on automated peritoneal dialysis (APD) worldwide, in long-standing as well as in more recently established peritoneal dialysis (PD) programs. A better understanding of the technique, paralleled by progress in involved technology, sustained this growth to the point that APD has become the prevalent mode of PD delivery in most high-income countries. While APD is now regarded to be at least as efficient as continuous ambulatory peritoneal dialysis (CAPD) with regard to major biomedical outcomes, its impact on patient-reported outcomes has been less investigated. This paper reviews the main outcomes of APD from a clinical point of view and from the person on dialysis perspective.
\end{abstract}

Keywords: cycler, intraperitoneal pressure, quality of life, prescription, tidal, ultrafiltration

\section{Introduction}

Patients requiring kidney replacement therapy (KRT) have a reduced health perception since they are chronically dependent from a life-saving procedure. Even if most of them strive to maintain well-being, they may suffer physical and psychological impairment that negatively affects their quality of life (QoL). Moreover, younger dialysis patients need to have free daytime for working both because they must do it and because employment is the better way to increase their well-being and QoL. ${ }^{1}$ When receiving understandable, unbiased information about (KRT) modality options, nearly half of the incident patients choose home PD, and the majority of them prefer APD. ${ }^{2}$ The main reason for this is the possibility to limit the burden of the dialysis procedure to nighttime, so minimizing restrictions to previous usual daytime activities of both the patient and/or the caregiver. Nevertheless, PD is used less than expected and prevalence is low worldwide, around $11 \% .^{3}$ This is because socioeconomic consideration, such as reimbursement policy, and staff confidence with PD influence the selection of dialysis modality and, in most of the cases, the patient's preference is not fully investigated. ${ }^{4}$ When PD is chosen and timely started, many clinicians opt for incremental manual peritoneal dialysis with one or two exchanges a day, switching to APD when an increase in PD dose becomes necessary, in order to preserve dialysis-free daytime, while others start directly with nightly APD (NIPD). ${ }^{5}$ Lifestyle reasons make APD the preferred modality in many developed countries. Its success, however, has been possible only thanks to the technological improvement of the cyclers.
Correspondence: Alessandro Domenici Department of Clinical and Molecular Medicine, "Sapienza" University, Sant'Andrea Hospital, Nephrology and Dialysis Unit, via di Grottarossa 1035 1039, Rome, 00189, Italy

Tel +39633775967

Fax +39633775320

Email adomenici@ospedalesantandrea.it 


\section{Historical Background}

The first apparatus conceived for PD delivery was first used in human by Boen et al in Seattle ${ }^{6}$ and subsequently by Lasker et $\mathrm{al}^{7}$ to treat patients with acute kidney injury (AKI). Further refinement of those devices subsequently allowed treatment of ESKD patients, and promoted a relative diffusion of intermittent APD. The use of APD was limited at that time to in-hospital treatment of ESKD patients 2-3 times a week for 8-10 hours per session, or to longer home treatments once a week. Uremic syndrome was only partially mitigated with those schedules and clinical results were quite poor, so that, with the advent of CAPD, APD was largely abandoned. In the late 1980s, renewed interest for APD was promoted by the commercialization of new machines, capable to pre-warm and quantitate peritoneal dialysis fluid (PDF) from apposite large volume bags. A milestone occurred in 1994 when, thanks to the improvement of hardware components, a compact and portable cycler, $12 \mathrm{~kg}$ in weight was developed. In the following version, a $2 \mathrm{Mb}$ data card was also provided, whose function was to transfer patient's prescription and to collect treatments reports (up to 60 days). The memory card allowed, for the first time, to monitor home therapy, including problems that patients may have during treatment, and compliance.

Subsequent diffusion of cycler-assisted PD provided evidence of potential advantages over CAPD in terms of prescription flexibility and rehabilitation issues, making APD the KRT of choice for children, actively working adults and older, frail patients dependent on a caregiver. Since then, APD has gained growing popularity, until becoming the prevalent mode of home PD delivery in most high-income countries. ${ }^{8}$ Rapid progress in technology has allowed great improvement in devices for APD in terms of safety issues, programming potential and portability. Last generation cyclers incorporate intra-peritoneal pressure (IPP) control, (pump-driven) inflow/outflow rate sensor, possibility to vary PDF composition, volume and dwell duration on a cycle by cycle basis during a session, giving room to better comfort for patients and caregivers while enhancing dialytic efficiency. ${ }^{9}$ Technological innovation has also made remote monitoring possible, with the advantage of closer, real time follow up. It has been found to improve patient's adherence to prescribed therapy, to enhance timely recognition of complications and proactive intervention, to reduce patient's unscheduled access to the hospital and to promote dialysis adequacy. ${ }^{10,11}$ These instruments have been well accepted by patients and their family. ${ }^{12}$ APD effectiveness has been recently reported also in particular clinical settings such as urgent PD start ${ }^{13}$ and treatment of acute kidney injury (AKI). ${ }^{14}$

\section{APD Prescription}

APD prescription entails basic orders such as duration of the session, total PDF volume used, number of cycles (inflow, dwell and outflow), dwell duration, intraperitoneal fill volume (IPV), total or fractional (tidal) exchange volume at each cycle, PDF composition with regard to glucose and calcium concentration and type of buffer (lactate or bicarbonate). Duration of nightly APD is usually tailored to patient's usual sleep time. The total PDF volume prescribed, as its composition, is dictated by target solute clearance and ultrafiltration (UF) volume. Increasing the PDF flow rate (ie, the "dose" of APD) enhances both solute clearance and UF, until an individual plateau is reached, beyond which no further increase is observed. This is because increase of total PDF volume per night means higher number of cycles, which translates in more treatment time spent on lower efficacy inflow/ outflow phases at the expenses of reduced dwell time. The PDF flow at plateau is higher for patients with medium to high peritoneal transport characteristics, and with the use of a tidal modality, while is reached earlier with a medium-low transport status and a nontidal modality. Measurement of the individual IPV/IPP curve allows to identify the highest tolerated IPV, which promotes efficiency while preventing overfill complaints and associated complications. Some of the last generation APD cyclers allow to vary IPV, PDF composition and dwell time on a cycle to cycle basis, offering the opportunity for an individualized optimization of the treatment. Furthermore, they incorporate flow sensors able to perceive the abrupt, persistent reduction in outflow rate (so called "breakpoint" or transition point) so that, instead of spending more time draining a small PDF volume, the cycler moves to the inflow phase of the next cycle (flexpoint), thus saving time for full volume dwell and theoretically improving dialytic efficiency. While promising, however, the efficacy of these features in real world clinical practice has not yet been clearly demonstrated in good quality trials to date.

\section{Clinical Outcomes}

In the last three decades APD has been regularly compared with CAPD in terms of incidence of infectious 
complications, residual kidney function (RKF) maintenance, small solute clearances and fluid balance target attainment, cost, quality of sleep, rehabilitation and quality of life (QoL), technique success and patient's survival. ${ }^{15}$

\section{Infectious Complications}

In the late 1990s, a lower incidence of peritonitis was consistently reported in patients on APD in comparison to those on CAPD, and possibly attributed to the minor number of connections/disconnections (ie, less opportunity for touch contamination) needed in APD. ${ }^{16}$ Later on, the overall incidence of PD-related peritonitis substantially declined thanks to the improvement of connection devices and the subsequent advent of no-disconnect systems, and no difference has been noted anymore between CAPD and APD in recent cohorts. ${ }^{17}$

\section{Residual Kidney Function Maintenance}

With APD diffusion, a number of papers reported a faster decline of RKF in patients on APD, and suggested intermittency of treatment could be a factor. Further large studies were less consistent, and even when a difference was found, it appeared to be small. ${ }^{18}$ According to published evidence, at present a possibly longer preservation of RKF is not regarded as decisive for the PD modality choice. Whenever an elective planned PD initiation is feasible and RKF (averaged urea and creatinine urinary clearances) is $>8 \mathrm{~mL} / \mathrm{min}$, PD may well be started with a single overnight, manually performed, exchange. ${ }^{19}$ This strategy associates low cost and the least intrusive transition to KRT, with the additional benefit the patient and/or the caregiver learn how to perform manual PD exchanges, an ability useful to increase flexibility of their life on PD and to cope with possible complications and clinical needs. In the author's experience, patients may continue with this low dose PD schedule for a minimum of 4-6 months to even several years, depending on the RKF course. When a single daily manual exchange is felt to be not enough, the option to switch to nightly APD should be offered, and about 2 patients out of 3 will choose it.

\section{Small Solute Clearance and Ultrafiltration (UF) Adequacy}

Prescription power of CAPD has long been established, and clinically confirmed, by satisfactory results achievable in most patients even when RKF is lost. Due to the short dwell time typical of nightly APD (NIPD), solute clearance and fluid removal are quite different and more heavily influenced by the patient's peritoneal transport status than in CAPD. While transperitoneal diffusion of small molecules such as urea is relatively fast, that of larger molecules such as creatinine, phosphorus, urate and sodium is much slower. As a consequence, NIPD (APD without day dwell) may achieve urea $\mathrm{KTp} / \mathrm{V}$ higher than CAPD, but creatinine and phosphate clearance and sodium $\left(\mathrm{Na}^{+}\right)$removal are usually substantially lower than those of CAPD, especially in patients with slow transport status. When RKF declines, NIPD alone may be not sufficient to reach presently recommended minimum adequacy targets, and one or more day dwells become necessary. On the other side, adequate UF volume is usually attained with NIPD, even in fast transporters experiencing difficult fluid balance with CAPD. On a clinical ground, there is general consensus on a preferential indication for APD in fast transporters. ${ }^{20,21}$ Moreover, UF efficiency, ie UF volume for grams of glucose absorbed, is nearly double that of CAPD, depending on peritoneal membrane transport status. Phosphate clearance is about $30 \%$ lower compared to full-dose CAPD, ${ }^{22}$ but dietary counseling, maintenance of RKF and chelating agents play a much more important role in counteracting hyperphosphatemia in PD patients. Sodium removal is usually lower with NIPD than with CAPD, but it approaches $70-100 \mathrm{mmol}$ per treatment in patients with no $\mathrm{RKF}^{23}$ making a sustainable dietary restriction usually enough to maintain balance. When large UF volume is needed, however, sodium sieving may cause hypernatremia, thirst and difficult fluid balance. The availability of lower $\mathrm{Na}^{+}$concentration PDF for APD would probably help here. ${ }^{24}$ Clinical advantages and drawbacks of APD when compared to CAPD are summarized in Table 1.

\section{Cost}

Direct cost of APD is about $30 \%$ higher than that of a 4 daily exchange CAPD schedule, with wide discrepancies between different countries and geographic areas. ${ }^{25}$ In western Europe, one 5-liter neutral $\mathrm{pH}$, low glucose degradation product (GDP) bag for APD costs about $20 \%$ more than one similar 2-liter CAPD bag and about 20\% less than a 2-liter polyglucose bag. Despite this, the majority of APD patients are prescribed a day-long polyglucose dwell, while NIPD dose (PDF volume per night) increasing is rarely pursued. Economic pressure did not promote research for the online production of PDF at the point of care, a potentially cost-saving development able to 
Table I Clinical and Biomedical Advantages and Drawbacks of APD Compared to CAPD

\begin{tabular}{|c|c|}
\hline \multicolumn{2}{|l|}{ Advantages } \\
\hline Larger prescription flexibility & $\begin{array}{l}\text { APD allows tailored personalization of fill volume, dwell time and glucose concentration each } \\
\text { cycle. Tidal and flex point allow to reduce time spent for low volume drainage }\end{array}$ \\
\hline Higher UF efficiency & Shorter dwell time compared to CAPD enhance UF, especially in fast transporters \\
\hline $\begin{array}{l}\text { Less frequent intraperitoneal pressure-related } \\
\text { complications }\end{array}$ & $\begin{array}{l}\text { Fine personalization of fill volume and UF every cycle may reduce abdomen overfilling } \\
\text { Lower IPP for any given IPV in supine position }\end{array}$ \\
\hline Lower incidence of PD-related peritonitis & $\begin{array}{l}\text { Less maneuvers may reduce the risk of touch contamination. However, with the introduction of } \\
\text { modern connection systems there seem to be no more differences between CAPD and APD }\end{array}$ \\
\hline $\begin{array}{l}\text { Better preserved peritoneal membrane } \\
\text { ("dry day", "peritoneal rest") }\end{array}$ & $\begin{array}{l}\text { Peritoneal rest may longer preserve the function of human peritoneal mesothelial cells. } \\
\text { Restoration of ultrafiltration has been described after peritoneal rest of a few months }\end{array}$ \\
\hline Easier dialytic dose increasing & $\begin{array}{l}\text { Increased volume per night or adding a daily exchange infused and drained by the cycler } \\
\text { (maneuver-free day dwell) may have low impact on patient's lifestyle }\end{array}$ \\
\hline Longer permanence on home PD & CCPD maximizes PD efficiency, assisted APD requires less daily visits \\
\hline Remote monitoring & $\begin{array}{l}\text { Real time bidirectional communication permits verify compliance, monitor treatment results and } \\
\text { timely change prescription according to clinical needs, reducing in-person visits }\end{array}$ \\
\hline \multicolumn{2}{|l|}{ Drawbacks } \\
\hline Lower phosphate and sodium removal & $\begin{array}{l}\text { Clearance of medium size molecules is lower in APD compared to CAPD because of short dwell } \\
\text { time. This effect may be mitigated by CCPD modality, possibly with icodextrin in the long dwell }\end{array}$ \\
\hline Faster decline of residual kidney function & It may be related to the intermittency of NIPD \\
\hline
\end{tabular}

Abbreviations: APD, automated peritoneal dialysis; CAPD, continuous ambulatory peritoneal dialysis; PD, peritoneal dialysis; UF, ultrafiltration; IPP, intraperitoneal pressure; IPV, intraperitoneal volume; CCPD, continuous cycling peritoneal dialysis; NIPD, nightly intermittent peritoneal dialysis.

substantially enhance APD efficiency, while dramatically reducing home space occupied by storage of PD supplies. A more meaningful analysis of APD cost would require inclusion of indirect costs and productivity rehabilitation issues, which probably largely compensate the direct cost gap with CAPD. ${ }^{26}$ As such, this evaluation is largely subject to regional health economy differences worldwide. In most low and low-middle gross domestic product (GDP) countries the offer of APD is still cost-restricted.

\section{Quality of Sleep, Rehabilitation and Quality of Life}

While early studies reported, as expected, worse sleep quality in patients on nightly APD, more recent ones found no difference or even reported a lower incidence of sleep apnea. ${ }^{27}$ Although modern cyclers are quite silent, quality of sleep of patients in APD may be negatively affected by the alarms raised during treatment. Sometimes patients report they stay awake because of the fear of alarms. Moving to a tidal prescription or preferring flexpoint may be helpful in reducing alarms when due to slow flow during the last part of drainage.

Interestingly, a recent small study found sleep quality to be associated with RKF rather than modality in PD patients. ${ }^{28}$ By no doubt, sleep quality is often heavily affected in ESKD patients, whatever the KRT modality, and is highly prioritized by patients, their family and caregivers. ${ }^{29}$ More extensive investigation is needed in this area.

Because of a less time demanding workload, APD may offer potential lifestyle benefits to the patient and his/her caregiver. Education attendance, job maintenance, active social life and leisure time are easier than with CAPD. Several studies show superior employment frequency among patients on APD, but this is largely because younger persons who have a job prefer APD over CAPD. ${ }^{30}$ Using validated measures of health-related quality of life (HRQoL), some studies reported a better QoL in patients on APD compared to those on CAPD, while others show comparable scores, with gradual improvement over time, more evident in patients on CAPD. ${ }^{31,32}$ 
A rapidly increasing percentage of patients who start KRT is old, frail, comorbid and dependent. In this scenario, the uptake of home PD is limited by the frequent unavailability of a family caregiver. ${ }^{2}$ To overcome this barrier, some countries have adopted and funded assistedPD (aPD) programs, which involve a trained nurse visiting patients at home to assist them for the dialytic procedure. ${ }^{33}$ PD utilization increased to some extent where aPD has been offered, and clinical outcomes and QoL appears to be at least as good as those of matched patients on in-center hemodialysis (iHD). ${ }^{34-36}$

\section{Technique Success and Patient Survival}

APD has the potential to serve patients at least as long as CAPD, and even longer in patients with no RKF and fast transport status, as well as those being (or near to become) dependent on a caregiver. Published studies on technique and patient survival with APD in comparison with CAPD produced conflicting results. Several papers found longer PD success with APD,${ }^{37}$ others did not, ${ }^{38}$ and due to the retrospective observational nature of these studies, the odds for potential confounding is high. The same holds true for patient survival, which has been reported as possibly longer with APD than with CAPD in some observational cohort studies, similar to that of CAPD patients in others. ${ }^{39}$ At present, PD modality choice in the individual patient should not rely upon the expectations of a longer PD success and/or patient survival.

\section{Patient's Values and Perceived Outcomes}

In recent years major attention has increasingly been paid to the patient's perspective of the goal of therapy, health-related quality of life (HRQoL) and patient reported outcomes (PROs). Research and guideline focus is moving away from classic biomedical adequacy targets toward a person-centered definition of treatment goals, tailored to the overall individual capability, beliefs, spiritual values, expectations and needs, of either the patient and his/her family and caregiver. Shared decision-making is now regarded as an extension of informed consent, potentially capable of promoting at the same time satisfactory clinical outcome and better quality of life and rehabilitation. Intuitively, this process holds particularly relevant to the care pathway of chronic debilitating diseases such as dialysis-requiring ESKD. ${ }^{40,41}$

The reason why a person in need of dialysis chooses home PD as the modality is usually its less intrusive impact on lifestyle and the possibility to self-manage therapy. Individuals choosing PD show high desire for control and are confident in taking responsibility for their therapy. ${ }^{42}$ Patient/caregiver perceived advantages and drawbacks of APD are summarized in Table 2.

For most patients nightly APD appears an attractive option for lifestyle purposes, being less disruptive of the usual daily activities. A few patients present machine phobia, and appear reluctant to be linked to the cycler the whole night. While safe temporary disconnection from the cycler is possible,

Table 2 Patient's Perceived APD Advantages and Drawbacks

\begin{tabular}{|l|l|}
\hline \multicolumn{2}{|l|}{ Advantages } \\
\hline Dialytic procedure limited to nighttime (NIPD): free daytime activity, ie school attendance, job maintenance, social life, leisure time \\
\hline Empty abdomen during the day: avoid sense of fullness, no body image distortion, reduced hernias and back pain, less restriction of physical activity \\
\hline Minimal burden for dialysis dose increase: increase PDF volume per night or maneuver-free day dwell managed by the cycler \\
\hline Drawbacks & Suggestions \\
\hline To be tied and dependent on a machine & $\begin{array}{l}\text { Teach safe temporary disconnection from the cycler. Confidence with the procedure may be } \\
\text { useful to overcome this barrier }\end{array}$ \\
\hline Possibly disrupted/disturbed sleep & Modify prescription to reduce alarms \\
\hline $\begin{array}{l}\text { Home space taken up for the adequate storage of } \\
\text { large volume of PD supplies }\end{array}$ & Provide fractionated more frequent deliveries \\
\hline Cycler or power failure & $\begin{array}{l}\text { Battery can last from half to } 2 \text { hours. In case of cycler failure, rapid substitution is guaranteed. } \\
\text { Manual exchanges can be done as an alternative during temporary unavailability of cycler }\end{array}$ \\
\hline Strength needed to lift and handle large PDF bags & Help by caregiver/assistant \\
\hline
\end{tabular}

Abbreviations: APD, automated peritoneal dialysis; NIPD, nightly intermittent peritoneal dialysis; PDF, peritoneal dialysis fluid; PD, peritoneal dialysis. 
reassurance and confidence may be useful to overcome this barrier. Cycler or power failure are easily managed by adequately trained patients and caregivers with temporary switch to manually performed PD exchanges, allowing time for power resumption or cycler technical assistance/substitution. After having tested cycler use at home, most patients are able to well tolerate nightly APD and report satisfactory sleep. Individually tailored, IPP-guided, fill volume prescription helps in delivering symptom-free treatment while promoting dialysis efficiency. Treatment outcome in details is recorded by the cycler and can be monitored remotely by the PD healthcare team. Whenever needed, modifications of the treatment schedule may be timely done as well. When an increase of the dialysis dose is needed, it is more easily managed with APD than with CAPD. While adding an additional daily manual exchange is reluctantly accepted by the patient, to connect one bag more to the cycler represents a marginal burden to the patient and caregiver. When a "dry day" NIPD (ie, no intraperitoneal PDF dwell during daytime) is felt not to be enough, day dwell may be managed with the help of the cycler, which infuses the desired volume of PDF at the end of the nightly treatment and drains it upon starting the next one. In this setting, the use of icodextrin avoids any additional maneuver during daytime, and has thus become popular and widely adopted. Some resilient, coping patients are motivated enough to incorporate even a midday manual exchange into their dialytic schedule (configuring what is called continuous cycling peritoneal dialysis, CCPD), in order not to switch to incenter HD. PD supplies for APD are bulky, and adequate space for storage at home may be problematic for a consistent percentage of concerned persons. ${ }^{43}$ Fractionated, more frequent delivery of PD supplies may help, while online production of PDF at home would greatly minimize this inconvenience. The possibility to have an empty abdomen during the day obviates body image issues, avoids sense of fullness and facilitates physical activity, while preventing or alleviating back pain and abdominal hernia complications.

Recognition of the patient's expectations, needs and values represents the first step for a meaningful education and a true shared-decision making process. Personcentered care means reasonable, realistic clinical outcome targets to be aligned with the individual values of the concerned person and his/her family and caregiver. Patient-reported outcome measures (PROMs) can provide valuable information that can help with the care of patients on APD. However, given the large number of measures available, it is unclear which PROMs are suitable for use in research or clinical practice. A Patient-Reported
Outcomes Measurement Information System (PROMIS) is currently being evaluated in some geographies. ${ }^{44-48}$

\section{Conclusion}

APD is acknowledged to be a valid KRT modality, being particularly attractive for younger pediatric patients, working-age adults and older, frail persons dependent on a caregiver. It favorably compares with other KRT modalities by promoting life participation and social function, the most prioritized PROs. Further technological improvement, remote monitoring, person/caregiver-centered KRT modality choice and cost reduction might promote its use further.

\section{Disclosure}

The authors declare no conflicts of interests with regard to the content and the publication of this paper.

\section{References}

1. Brown EA, Zhao J, McCullough K, et al. Burden of kidney disease, health-related quality of life, and employment among patients receiving peritoneal dialysis and in-center hemodialysis: Findings from the DOPPS program. Am J Kidney Dis. 2021; 78 (4):481-500. doi:10.1053/j.ajkd.2021.

02.327

2. Jager K, Korevaar J, Dekker F, et al. The effect of contraindications and patient preference on dialysis modality selection in ESRD patients in the Netherlands. Am J Kidney Dis. 2004;43:891-899. doi:10.1053/j.ajkd.2003.12.051

3. Cho Y, Bello AK, Levin A, et al. Peritoneal dialysis use and practice patterns: an international survey study. Am J Kidney Dis. 2021;77 (3):315-325. doi:10.1053/j.ajkd.2020.05.032

4. Van Biesen W, Lameire N. Increasing peritoneal dialysis initiation worldwide: 'there are none so blind as those who will not see'. Nephrol Dial Transplant. 2020;35(9):1458-1461. doi:10.1093/ndt/ gfaa024

5. Neri L, Viglino G, Marinangeli G, et al. Incremental start to PD as experienced in Italy: results of censuses carried out from 2005 to 2014. J Nephrol. 2017;30(4):593-599. doi:10.1007/s40620-017-0403

6. Boen ST, Mion CM, Curtis FK, Shilipetar G. Periodic peritoneal dialysis using the repeated puncture technique and an automatic cycling machine. Trans Am Soc Artif Intern Organs. 1964;10: 409-414.

7. Lasker N, McCauley EP, Passarotti CT. Chronic peritoneal dialysis. Trans Am Soc Artif Intern Organs. 1966;12:94-97.

8. Wang AY, Zhao J, Bieber B, et al; PDOPPS Dialysis Prescription and Fluid Management Working Group. International comparison of peritoneal dialysis prescriptions from the peritoneal dialysis outcomes and practice patterns study (PDOPPS). Perit Dial Int. 2020;40 (3):310-319. PMID: 32063209. doi: 10.1177/0896860819895356

9. Chaudhry RI, Golper TA. Automated cyclers used in peritoneal dialysis: technical aspects for the clinician. Med Devices. 2015;8:95-102. PMID: 25653566; PMCID: PMC4311757. doi:10. 2147/MDER.S51189

10. Milan Manani S, Baretta M, Giuliani A, et al. Remote monitoring in peritoneal dialysis: benefits on clinical outcomes and on quality of life. J Nephrol. 2020;33(6):1301-1308. PMID: 32779144; PMCID: PMC7416995. doi: 10.1007/s40620-020-00812-2 
11. Amici G, D'Angela D, Lo Cicero A, Romanini D, Martino FK, Spandonaro F. Pilot health technology assessment study: organizational and economic impact of remote monitoring system for home automated peritoneal dialysis. Int Urol Nephrol. 2021;53(9):1933-1940. PMID: 33675485. doi:10.1007/s11255-02102816-8

12. Walker RC, Tong A, Howard K, Darby N, Palmer SC. Patients' and caregivers' expectations and experiences of remote monitoring for peritoneal dialysis: a qualitative interview study. Perit Dial Int. 2020;40(6):540-547. doi:10.1177/0896860820927528

13. Javaid MM, Khan BA, Subramanian S. The modality of choice, manual or automated, for urgent start peritoneal dialysis. Clin Kidney J. 2019;12(3):443-446. doi:10.1093/ckj/sfz008

14. Al-Hwiesh A, Abdul-Rahman I, Finkelstein F, et al. Acute kidney injury in critically ill patients: a prospective randomized study of tidal peritoneal dialysis versus continuous renal replacement therapy. Ther Apher Dial. 2018;22(4):371-379. doi:10.1111/17449987.12660

15. Bieber SD, Burkart J, Golper TA, Teitelbaum I, Mehrotra R. Comparative outcomes between continuous ambulatory and automated peritoneal dialysis: a narrative review. Am J Kidney Dis. 2014;63(6):1027-1037. PMID: 24423779; PMCID: PMC4300314. doi:10.1053/j.ajkd.2013.11.025

16. Rodriguez-Carmona A, Perez Fontan M, Garcia Falcon T, Fernandez Rivera C, Valdes F. A comparative analysis on the incidence of peritonitis and exit-site infection in CAPD and automated peritoneal dialysis. Perit Dialysis Int. 1999;19(3):253-258. doi:10.1177/089 686089901900311

17. Lan PG, Johnson DW, McDonald SP, et al. The association between peritoneal dialysis modality and peritonitis. Clin J Am Soc Nephrol. 2014;9(6):1091-1097. doi:10.2215/CJN.09730913

18. Michels WM, Verduijn M, Grootendorst DC, et al. Decline in residual renal function in automated compared with continuous ambulatory peritoneal dialysis. Clin J Am Soc Nephrol. 2011;6(3):537-542. doi: $10.2215 / \mathrm{CJN} .00470110$

19. Foggensteiner L, Baylis J, Moss H, Williams P. Timely initiation of dialysis-single-exchange experience in 39 patients starting peritoneal dialysis. Perit Dial Int. 2002;22(4):471-476. doi:10.1177/0896860 80202200405

20. Yang X, Fang W, Bargman JM, Oreopoulos DG. High peritoneal permeability is not associated with higher mortality or technique failure in patients on automated peritoneal dialysis. Perit Dialysis Int. 2008;28(1):82-92. doi:10.1177/089686080802800114

21. Johnson DW, Hawley CM, McDonald SP, et al. Superior survival of high transporters treated with automated versus continuous ambulatory peritoneal dialysis. Nephrol Dial Transplant. 2010;25(6):19 73-1979. doi:10.1093/ndt/gfp780

22. Debowska M, Gomez R, Pinto J, Waniewski J, Lindholm B. Phosphate clearance in peritoneal dialysis. Sci Rep. 2020;10 (1):17504. doi:10.1038/s41598-020-74412-2

23. Borrelli S, La Milia V, De Nicola L, et al. Sodium removal by peritoneal dialysis: a systematic review and meta-analysis. J Nephrol. 2019;32(2):231-239. doi:10.1007/s40620-018-0507-1

24. Rutkowski B, Tam P, van der Sande FM, et al. Residual renal function and effect of low-sodium solution on blood pressure in peritoneal dialysis patients. Perit Dial Int. 2019;39(4):335-343. doi:10.3747/pdi.2018.00120

25. Karopadi AN, Mason G, Rettore E, Ronco C. The role of economies of scale in the cost of dialysis across the world: a macroeconomic perspective. Nephrol Dial Transplant. 2014;29(4):885-892. doi:10.1093/ ndt/gft528

26. Julián-Mauro JC, Cuervo J, Rebollo P, Callejo D. Employment status and indirect costs in patients with renal failure: differences between different modalities of renal replacement therapy. Nefrologia. 2013;33(3):333-341. doi:10.3265/Nefrologia.pre2012. Dec. 11767
27. Tang SC, Lam B, Ku PP, et al. Alleviation of sleep apnea in patients with chronic renal failure by nocturnal cycler-assisted peritoneal dialysis compared with conventional continuous ambulatory peritoneal dialysis. J Am Soc Nephrol. 2006;17(9):2607-2616. doi:10.1681/ ASN.2005090936

28. Lanis A, Kerns E, Hu SL, et al. Residual renal function affects severity of sleep apnea in peritoneal dialysis: a pilot study. Lung. 2018;196(4):425-431. doi:10.1007/s00408-018-0127-5

29. De Silva I, Evangelidis N, Hanson CS, et al. Patient and caregiver perspectives on sleep in dialysis. J Sleep Res. 2021;30(4):e13221. doi:10.1111/jsr.13221.

30. Julian Mauro JC, Molinuevo Tobalina JA, Sánchez González JC. Employment in the patient with chronic kidney disease related to renal replacement therapy. Nefrologia. 2012;32(4):439-445. doi:10.3265/Nefrologia.pre2012.Apr.11366

31. Jung HY, Jang HM, Kim YW, et al. Depressive symptoms, patient satisfaction, and quality of life over time in automated and continuous ambulatory peritoneal dialysis patients: a prospective multicenter propensity-matched study. Medicine. 2016;95(21):e3795. doi:10.1097/MD.0000000000003795

32. Yang F, Luo N, Lau T, Yu ZL, Foo MWY, Griva K. Health-related quality of life in patients treated with continuous ambulatory peritoneal dialysis and automated peritoneal dialysis in Singapore. Pharmacoecon Open. 2018;2(2):203-208. doi:10.1007/s41669-017-0046-z

33. Giuliani A, Karopadi AN, Prieto-Velasco M, Manani SM, Crepaldi C, Ronco C. Worldwide experiences with assisted peritoneal dialysis. Perit Dial Int. 2017;37(5):503-508. doi:10.3747/pdi.2016.00214

34. Boyer A, Solis-Trapala I, Tabinor M, Davies SJ, Lambie M. Impact of the implementation of an assisted peritoneal dialysis service on peritoneal dialysis initiation. Nephrol Dial Transplant. 2020;35 (9):1595-1601. doi:10.1093/ndt/gfz287

35. Iyasere O, Brown E, Gordon F, et al. Longitudinal trends in quality of life and physical function in frail older dialysis patients: A comparison of assisted peritoneal dialysis and in-center hemodialysis. Perit Dial Int. 2019;39(2):112-118. doi:10.3747/ pdi.2018.00086

36. Maierean SM, Oliver MJ. Health outcomes and cost considerations of assisted peritoneal dialysis: A narrative review. Blood Purif. 2021;1-5. doi:10.1159/000512839

37. Sun CY, Lee CC, Lin YY, Wu MS. In younger dialysis patients, automated peritoneal dialysis is associated with better long-term patient and technique survival than is continuous ambulatory peritoneal dialysis. Perit Dial Int. 2011;31(3):301-307. doi:10.3747/pdi.2010.00072

38. Beduschi Gde C, Figueiredo AE, Olandoski M, et al. Automated peritoneal dialysis is associated with better survival rates compared to continuous ambulatory peritoneal dialysis: a propensity score matching analysis. PLoS One. 2015;10(7):e0134047. doi:10.1371/ journal.pone. 0134047

39. Rabindranath KS, Adams J, Ali TZ, Daly C, Vale L, Macleod AM. Automated vs continuous ambulatory peritoneal dialysis: a systematic review of randomized controlled trials. Nephrol Dial Transplant. 2007;22(10):2991-2998. doi:10.1093/ndt/gfm515

40. Brown EA, Blake PG, Boudville N, et al. International society for peritoneal dialysis practice recommendations: Prescribing high-quality goal-directed peritoneal dialysis. Peritoneal Dialysis Int. 2020;40(3):244-253. doi:10.1177/0896860819895364

41. Blake PG, Brown EA. Person-centered peritoneal dialysis prescription and the role of shared decision-making. Perit Dial Int. 2020;40 (3):302-309. doi:10.1177/0896860819893803

42. Schellartz I, Ohnhaeuser T, Mettang T, Scholten N. The role of personal attitudes of control and responsibility for the uptake of peritoneal dialysis- a national survey among dialysis patients. $B M C$ Nephrol. 2021;22(1):107. doi:10.1186/s12882-021-02303-3

43. Sukul N, Zhao J, Fuller DS, et al. Patient-reported advantages and disadvantages of peritoneal dialysis: results from the PDOPPS. BMC Nephrol. 2019;20(1):116. doi:10.1186/s12882-019-1304-3 
44. Budhram B, Sinclair A, Komenda P, Severn M, Sood MM. A comparison of patient-reported outcome measures of quality of life by dialysis modality in the treatment of kidney failure: a systematic review. Can $J$ Kidney Health Dis. 2020;7:2054358120957431. doi:10.1177/2054358120957431

45. Manera KE, Johnson DW, Craig JC, et al. Patient and caregiver priorities for outcomes in peritoneal dialysis: Multinational nominal group technique study. Clin J Am Soc Nephrol. 2019;14(1):74-83. doi:10.2215/CJN.05380518

46. Griva K, Goh CS, Kang WCA, et al. Quality of life and emotional distress in patients and burden in caregivers: a comparison between assisted peritoneal dialysis and self-care peritoneal dialysis. Qual Life Res. 2016;25(2):373-384. doi:10.1007/s11136-015-1074-8
47. Manera KE, Johnson DW, Craig JC, et al. Establishing a core outcome set for peritoneal dialysis: Report of the SONG-PD (standardized outcomes in nephrology-peritoneal dialysis) consensus workshop. Am J Kidney Dis. 2020;75(3):404-412. doi:10.1053/j. ajkd.2019.09.017

48. Finkelstein FO, Foo MW. Health-related quality of life and adequacy of dialysis for the individual maintained on peritoneal dialysis. Perit Dial Int. 2020;40(3):270-273. doi:10.1177/ 0896860819893815

\section{Publish your work in this journal}

The International Journal of Nephrology and Renovascular Disease is an international, peer-reviewed open-access journal focusing on the pathophysiology of the kidney and vascular supply. Epidemiology, screening, diagnosis, and treatment interventions are covered as well as basic

science, biochemical and immunological studies. The manuscript management system is completely online and includes a very quick and fair peer-review system, which is all easy to use. Visit http://www.dovepress.com/testimonials.php to read real quotes from published authors. 